\title{
Transcriptome analysis of Bupleurum chinense focusing on genes involved in the biosynthesis of saikosaponins
}

\author{
Chun Sui, Jie Zhang, Jianhe Wei*, Shilin Chen, Ying Li, Jiesen Xu, Yue Jin, Caixiang Xie, Zhihui Gao, \\ Hongjiang Chen, Chengmin Yang, Zheng Zhang and Yanhong Xu
}

\begin{abstract}
Background: Bupleurum chinense DC. is a widely used traditional Chinese medicinal plant. Saikosaponins are the major bioactive constituents of $B$. chinense, but relatively little is known about saikosaponin biosynthesis. The 454 pyrosequencing technology provides a promising opportunity for finding novel genes that participate in plant metabolism. Consequently, this technology may help to identify the candidate genes involved in the saikosaponin biosynthetic pathway.

Results: One-quarter of the 454 pyrosequencing runs produced a total of 195, 088 high-quality reads, with an average read length of 356 bases (NCBI SRA accession SRA039388). A de novo assembly generated 24, 037 unique sequences (22, 748 contigs and 1, 289 singletons), 12, 649 (52.6\%) of which were annotated against three public protein databases using a basic local alignment search tool (E-value $\leq 1 \mathrm{e}-10$ ). All unique sequences were compared with NCBI expressed sequence tags (ESTs) (237) and encoding sequences (44) from the Bupleurum genus, and with a Sanger-sequenced EST dataset $(3,111)$. The 23, 173 (96.4\%) unique sequences obtained in the present study represent novel Bupleurum genes. The ESTs of genes related to saikosaponin biosynthesis were found to encode known enzymes that catalyze the formation of the saikosaponin backbone; 246 cytochrome P450 (P450s) and 102 glycosyltransferases (GTs) unique sequences were also found in the 454 dataset. Full length cDNAs of 7 P450s and 7 uridine diphosphate GTS (UGTs) were verified by reverse transcriptase polymerase chain reaction or by cloning using $5^{\prime}$ and/or $3^{\prime}$ rapid amplification of CDNA ends. Two P450s and three UGTs were identified as the most likely candidates involved in saikosaponin biosynthesis. This finding was based on the coordinate up-regulation of their expression with $\beta$-AS in methyl jasmonate-treated adventitious roots and on their similar expression patterns with $\beta$-AS in various $B$. chinense tissues.Conclusions

A collection of high-quality ESTs for $B$. chinense obtained by 454 pyrosequencing is provided here for the first time. These data should aid further research on the functional genomics of $B$. chinense and other Bupleurum species. The candidate genes for enzymes involved in saikosaponin biosynthesis, especially the P450s and UGTs, that were revealed provide a substantial foundation for follow-up research on the metabolism and regulation of the saikosaponins.
\end{abstract}

\section{Background}

Bupleurum chinense DC., a perennial herb native to China, belongs to the Umbelliferae family and the genus Bupleurum L. This herb is used worldwide for medicinal purposes, but is especially common in China, Japan, and

\footnotetext{
* Correspondence: jhwei@implad.ac.cn

Institute of Medicinal Plant Development (IMPLAD), Chinese Academy of Medical Sciences \& Peking Union Medical College, No. 151, Malianwa North Road, Haidian District, Beijing 100193, China
}

South Korea [1]. In traditional Chinese medicine, the roots of B. chinense and other Bupleurum species are known as Chinese thorowax roots (Radix bupleuri), or "chaihu" in Chinese. For more than 2, 000 years these roots have been used for their anti-inflammatory, antipyretic, and anti-hepatotoxic effects in the treatment of common colds, fever, influenza, hepatitis, malaria, and menoxenia [2,3]. The major bioactive components of Radix bupleuri are the saikosaponins (SSs), which

\section{Biomed Central}

(c) 2011 Sui et al; licensee BioMed Central Ltd. This is an Open Access article distributed under the terms of the Creative Commons Attribution License (http://creativecommons.org/licenses/by/2.0), which permits unrestricted use, distribution, and reproduction in any medium, provided the original work is properly cited. 
belong to the oleanane-type triterpene saponins. Although more than 75 monomer SSs have been isolated from Radix bupleuri $[4,5]$, only SS-a, SS-b2, SS-c, and SS-d have been pharmacologically examined [6-10], because of the low SS content (usually ca. $1 \% w / w$ in dried roots) [11]. Different monomer SSs have been reported to exhibit different predominant pharmacological effects. For example, among the SSs isolated from $B$. falcatum, SS-a and SS-d, but not SS-c, have anti-inflammatory activities [12]. Whereas SS-c has no correlation with cell growth inhibition, other SSs can inhibit cell growth, as well as induce cancer cell differentiation and apoptosis. Hence, SS-c may have the potential for therapeutic angiogenesis, but is unsuitable for cancer therapy [10]. Roots derived from various Bupleurum species such as B. chinense, B. scorzonerifolium, B. falcatum, and $B$. kaoi, have been widely used in various medicinal decoctions. The content and proportion of the monomer SSs are extremely diverse in these medicinal materials. The concentration and composition of the SSs in the roots is even more complex when studied in combination with diverse planting and harvesting environments and different management methods.

The ability to control the SS content of these medicinal materials by up-regulating the genes involved in the biosynthesis of the different SS monomers or by using bio-engineering techniques would greatly improve their reliability. To be able to attempt this, an understanding of SS biosynthesis is required [13]. The putative SS biosynthetic pathway in $B$. chinense is shown in Figure 1. This pathway is based on previous studies on the biosynthetic pathway of other triterpene saponins in some plant species $[14,15]$, as well as the pathways of SSs in B. falcatum [16] and B. kaoi [17]. The putative SS biosynthetic pathway initiates the isoprenoid pathway, mediates the cyclization of oxidosqualene, and then undergoes some modifications of oxidation, glycosylation, and other secondary transformations. Finally, the formation of the various monomer SSs is completed. The cDNA of $\beta$-amylase ( $\beta$-AS) that catalyzes the formation of $\beta$-amyrin has been cloned in B. kaoi [18], and two different cDNAs that may encode different isoforms of $\beta$-AS have been cloned in B. chinense. Recently, these clones have been characterized in our laboratory (unpublished). In our previous studies, the cDNAs that encode enzymes involved upstream of the SS biosynthetic pathway in $B$. chinense have also been cloned [19-21]]. The biosynthetic pathways of the other saponins are not fully understood. Similarly, the reactions downstream of the SS biosynthetic pathway after the cyclization of $\beta$-amyrin remain largely unknown. One proposal involves oxidization/hydroxylation and glycosyl transfer catalyzed by specific cytochrome P450s and uridine diphosphate (UDP) glycosyltransferases (UGTs), respectively $[14,22,23]]$. To date, no P450 or UGT genes involved in SS biosynthesis have been identified in $B$. chinense or in other SS-producing plants. To the best of our knowledge, only a few P450s and UGTs have been verified to be involved in the biosynthesis of triterpenoid saponins [24-28]; recent reviews in [29,30]]. This situation is inconsistent with the fact that saponins are widely distributed in plants [31]. The biosynthetic pathway of the saponins still has to be clarified.

Studies have shown that the sequencing and analysis of expressed sequence tags (ESTs), combined with genetic and phytochemical methods are effective tools for discovering novel genes in non-model plants [32-34]]. Some genes involved in natural product biosynthetic pathways have been identified via EST analyses [24-26,28,35,36]]. The 454 pyrosequencing technique, with its advantages of throughput, read length, and accuracy, can greatly accelerate the discovery of novel genes in non-model organisms [37-39]]. Candidate genes involved in the metabolic pathways of natural products have been identified using the 454 pyrosequencing technique. These natural products usually have diverse and important functions in plant growth, and are also invaluable as pharmaceuticals and agrochemicals. Examples of such important natural products include triterpene saponins in American ginseng [15] and Glycyrrhiza uralensis [40], flavonoids in Artemisia annua [41], alkaloids in Huperzia serrata and Phlegmariurus carinatus [42], and cyanogenic glucosides in Zygaena filipendulae [43]. The specific functions of the candidate genes reported in the abovementioned studies are still being validated. Even so, the 454 pyrosequencing is still the preferred choice for novel gene discovery, especially for members of known gene families.

In the present study, 195, 088 high-quality (HQ) reads from a cDNA library of $B$. chinense were obtained using the Roche GS FLX Titanium platform. The reads were assembled into 24, 037 unique sequences comprising 22, 748 contigs and 1, 289 singletons. Only 864 ESTs were identical with those derived from the 3, 111 ESTs generated in our previous study from a $B$. chinense root cDNA library using the Sanger sequencing method [19] and the Bupleurum sequences from NCBI. A total of 246 P450s and 102 glycosyltransferases (GTs) including 49 UGTs were screened. The assembled full-length cDNAs of the P450s and UGTs were verified. Several partial cDNAs of the P450s and UGTs were extended to full length by 5' and/or 3' rapid amplification of cDNA ends (RACE). The candidate P450s and UGTs that may participate in SS biosynthesis were screened via methyl jasmonate (MeJA) inducibility and tissue-specific expression pattern experiments. These P450s and UGTs will be the targets of further research on SS biosynthesis. 


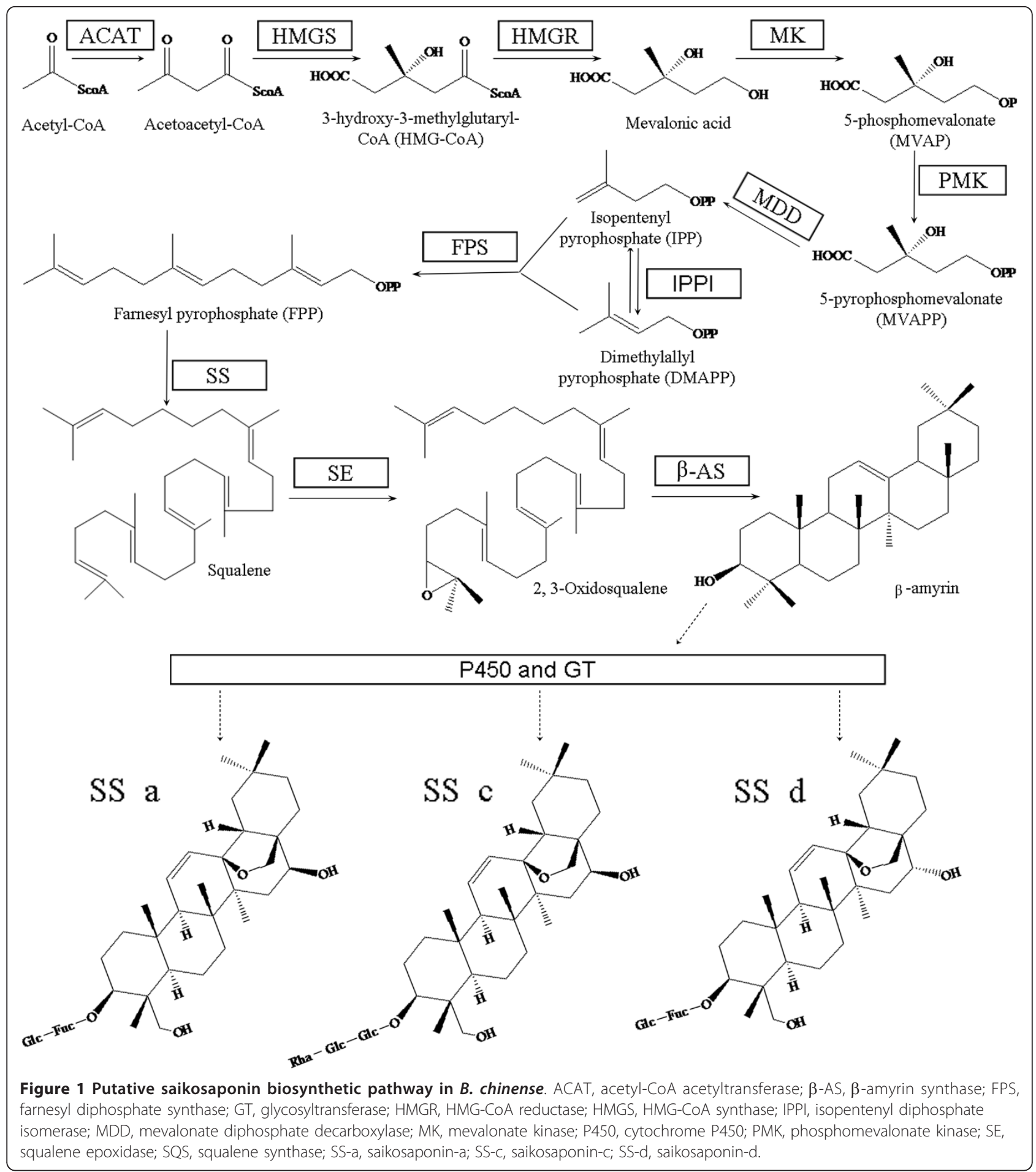

\section{Results}

\section{Sequencing and de novo assembly}

A one-quarter plate run using the 454 GS FLX Titanium platform was carried out on the cDNA that was generated by SMART technology from the equivalent pooled total RNA from $B$. chinense roots, germinating seeds, and seedlings. A total of 195, 088 HQ reads with an average sequence length of $356 \mathrm{bp}$ were obtained from 202, 126 raw reads after the initial quality filtering step [NCBI Short Read Archive, accession SRA039388]. The HQ reads were then assembled into 22, 748 contigs and 1, 289 singletons. The consensuses (contigs and 
Table 1 Summary of $B$. chinense 454 sequencing and assembly

\begin{tabular}{lllll}
\hline Items & $\begin{array}{l}\text { High-quality } \\
\text { reads }\end{array}$ & Contigs & Singletons & Consensuses \\
\hline Total number & 195088 & 22748 & 1289 & 24037 \\
$\begin{array}{l}\text { Total bases } \\
\text { (bp) }\end{array}$ & 69381890 & 13402551 & 439796 & 13842347 \\
$\begin{array}{l}\text { Average } \\
\text { length }\end{array}$ & 355.7 & 589.2 & 341.2 & 575.9 \\
$\begin{array}{l}\text { Range of } \\
\text { length }\end{array}$ & $50-711$ & $43-2756$ & $51-572$ & $43-2756$ \\
\hline
\end{tabular}

singletons), equal to $13.8 \mathrm{Mb}$ of sequence data, had an average length of $576 \mathrm{bp}$ with a range of 43-2, $756 \mathrm{bp}$. The sequencing and assembly statistics are shown in Table 1. The size distribution of the consensuses is shown in Figure 2.

\section{Functional annotation}

The assembled 24, 037 unique sequences were successively compared with the sequences in three major public protein databases (KEGG, Nr, and UniProt) using the basic local alignment search tool X (BLASTX) algorithm with an E-value cutoff of $<10^{-10}$. A total of 12, 649 unique sequences, accounting for $52.6 \%$ of the total unique sequences, were annotated (See additional file 1: Summary of the annotation of the 454 assembled unique $B$. chinense sequences). In our previous study, a total of 3, $111 \mathrm{cDNA}$ clones derived from a $B$. chinense root cDNA library were 5' single-pass-sequenced using the Sanger sequencing method [19]. In the present study, the unique sequences obtained via 454 pyrosequencing were compared with the 3,111 ESTs and with an additional 237 ESTs downloaded from GenBank. The unique sequences were also compared with the 44 Bupleurum protein-encoded sequences from GenBank.
Only 864 unique sequences from the 454 dataset overlapped with other ESTs. Therefore, the 454 dataset must contain thousands of novel genes for the genus Bupleurum (Figure 3).

A sum of 10, 734 (44.7\%) of the total unique sequences were further annotated based on their similarity with The Arabidopsis Information Resource (TAIR) proteins, before gene ontology (GO) terms were assigned. The categories of molecular function, biological process, and cellular component are shown in additional file 2: Functional annotations of the 454 unique sequences of $B$. chinense based on GO categories. A high percentage of the unique sequences were annotated to hydrolases, kinases, and transferases in the molecular function category. For the biological process category, a large number of genes were annotated to metabolic processes, response to abiotic or biotic stimulus, and response to stress. Hence, the 454 dataset should substantially aid the discovery of novel genes involved in the metabolism of SSs and other secondary natural products. A total of 10, 277 unique sequences were annotated using KEGG; 2,849 of them were related to metabolism, 36 to the metabolism of terpenoids polyketides, and 101 to the biosynthesis of other secondary metabolites (see additional file 3: Summary of metabolic pathway assignments of the 454 assembled unique sequences based on KEGG).

\section{Candidate genes related to SS backbone biosynthesis}

Similar to other triterpene glycoside pathways, the SS biosynthetic pathway is generally divided into three stages (Figure 1). The first stage is the formation of the isoprene units isopentenyl pyrophosphate (IPP) and dimethylallyl pyrophosphate (DMAPP), the second is the formation of the triterpene skeleton ( $\beta$-amyrin), and the third stage is the modification of the skeleton.

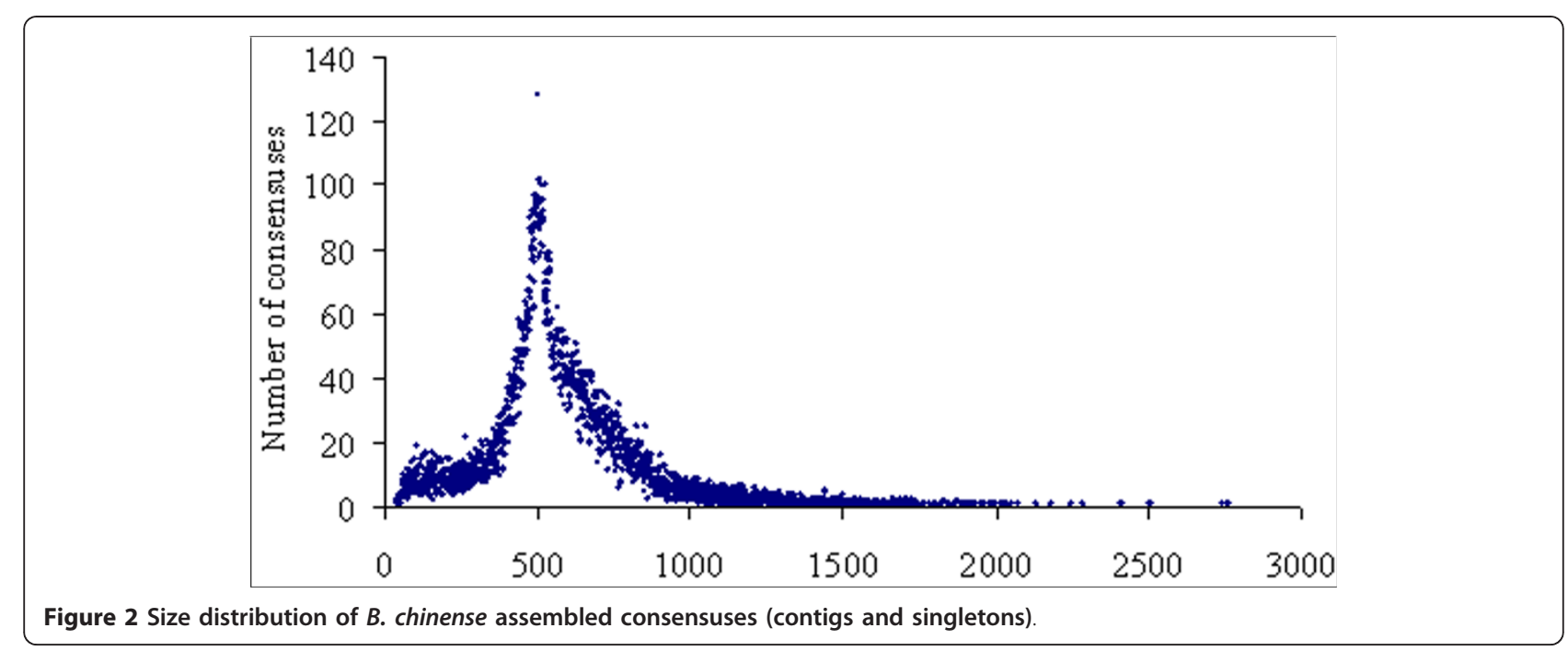




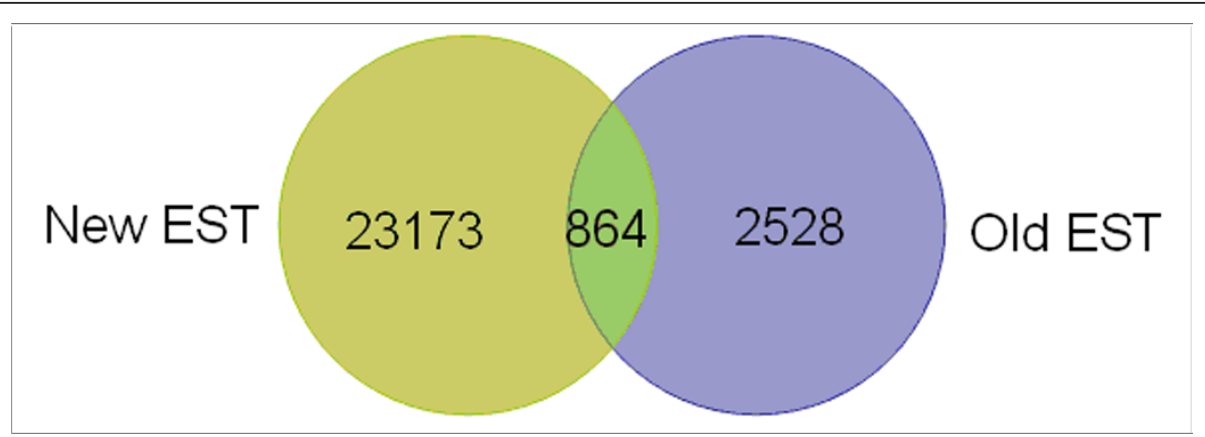

Figure 3 Comparison of the 454 EST dataset and other EST datasets obtained for the genus Bupleurum. The overlapping section represents the ESTs that are present in the two datasets.

Based on our current knowledge, no definite sequence features can be used to identify the specific P450s and UGTs involved in the modification of the triterpene skeleton. Therefore, the unique sequences that were annotated as responsible for the formation of the SS backbones in the first two stages of the pathway were first screened. As shown in Table 2, except for mevalonate-5-diphosphate decarboxylase (MDD), the putative genes that encode all the enzymes for the biosynthesis of SS backbones were found. Triterpenoids are generally considered to be formed in the cytoplasm via the mevalonate (MVA) pathway. However, recent reports have demonstrated that the cytosolic MVA pathway and the plastidic methylerythritol phosphate (MEP) pathway may mutually communicate through the regulatory role of isopentenyl diphosphate isomerase (IPPI), which maintains the appropriate levels of IPP and DMAPP in the cytoplasm and plastids [44,45]. Consequently, three putative genes that encode the enzymes involved in the MEP pathway were also searched for and found in the 454 dataset. These genes were DXP reductoisomerase (DXPS; EC 1.1.1.267), MEP cytidylyltransferase (MEPCT; EC 2.7.7.60), and 4-hydroxy-3methylbut-2-enyl-diphosphate synthase (GcpE/IspG; EC 1.17.7.1).

\section{Full-length CDNA cloning of P450s and UGTs}

The P450s constitute one of the biggest gene families in plant genomes, accounting for more than $1 \%$ of the total gene annotations in each plant species [22]. In our unique sequences, a total of 239 contigs and 7 singletons were annotated as $P 450$ s accounting for about $1.02 \%$ of the 24, 037 unique sequences (see additional file 4: Putative P450 and GT genes in the 454 dataset). According to the best-hit description in the annotation databases, the P450s encoded by these unique sequences were classified into 30 families and 44 subfamilies; four of the P450s were unclassified (see additional file 5: Summary of family classification of the annotated P450s from the 454 assembled unique sequences). Two annotated full-length $P 450$ s were verified by reverse transcriptase polymerase chain reaction (RT-PCR). Three P450s that had single terminals in the 454 dataset, and two P450s derived from our previous cDNA library, were extended by RACE PCR. As a result, seven fulllength $P 450$ s were obtained. The seven full-length $P 450$ s were further analyzed using BLASTX in NCBI. The deduced amino acid sequences were aligned with homologous Arabidopsis thaliana P450s and some functionidentified P450s from other species. A neighbor-joining tree was constructed based on the alignment (Figure 4).

Table 2 Numbers of annotated unique sequences and 454 reads involved in saikosaponin skeleton biosynthesis

\begin{tabular}{lllll}
\hline Enzyme code & Abbreviation & Enzyme name & Number of unique sequences & Number of 454 reads \\
\hline 2.3.1.9 & ACAT & Acetyl-CoA acetyltransferase & 4 & 10 \\
2.3.3.10 & HMGS & HMG-CoA synthase & 2 & 6 \\
1.1.1.34 & HMGR & HMG-CoA reductase & 1 & 2 \\
2.7.1.36 & MK & Mevalonate kinase & 1 & 2 \\
2.7.4.2 & PMK & Phosphomevalonate kinase & 1 & 1 \\
4.1.1.33 & MDD & Mevalonate-5-diphosphate decarboxylase & 0 & 0 \\
5.3 .3 .2 & PPI & Isopentenyl-PP isomerase & 1 & 1 \\
2.5.1.10 & FS & Farnesyl diphosphate synthase & 2 & 27 \\
2.5.1.21 & SQS & Squalene synthase & 6 & 30 \\
5.14 .99 .7 & SE & Squalene epoxidase & 1 & 1 \\
\hline
\end{tabular}




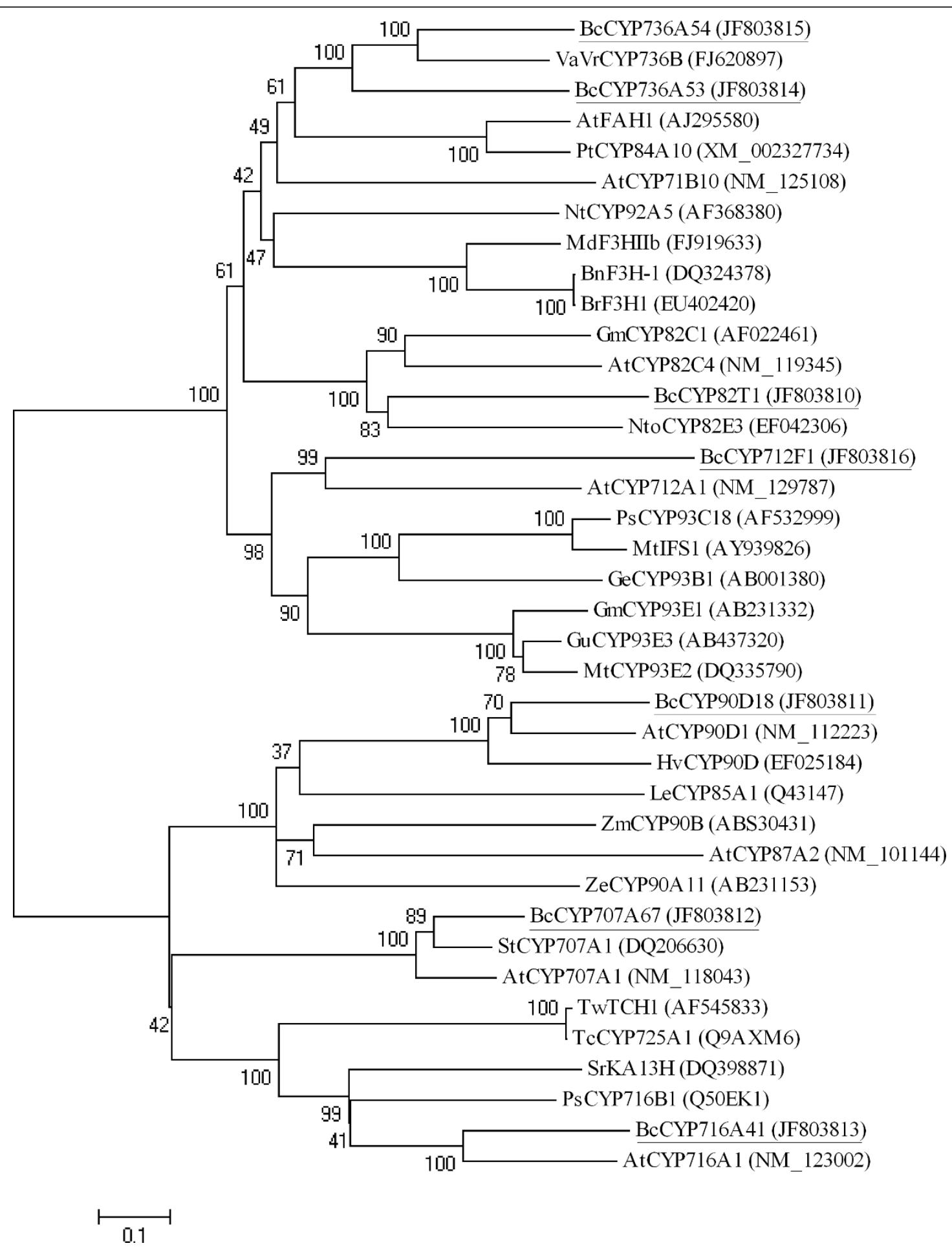

Figure 4 Neighbor-joining bootstrap tree of P450 sequences from B. chinense and from other plant species. Bootstrap values are shown as percentages. Sequences from B. chinense are underlined. The P450 sequences from B. chinense are the translated full-length sequences. At, Arabidopsis thaliana; Bc, Bupleurum chinense; Bn, Brassica napus; Br, Brassica rapa subsp. Campestris; Ge, Glycyrrhiza echinata; Gm, Glycine max; Gu, Glycyrrhiza uralensis; Hv, Hordeum vulgare subsp. Vulgare; Le, Lycopersicon esculentum; Lj, Lotus japonicus; Md, Malus x domestica; Mt, Medicago truncatula; Nt, Nicotiana tabacum; Nto, Nicotiana tomentosiformis; Ps, Pisum sativum; Pt, Populus trichocarpa; Sr, Stevia rebaudiana; St, Solanum tuberosum; Tc, Taxus cuspidate; Tw, Taxus wallichiana var. chinensis; VaVr, Vitis arizonica $\times$ Vitis rupestris; Ze, Zinnia elegans; Zm, Zea mays. 
The seven P450s were named by Nelson [46] as BcCYP82T1, BcCYP90D18, BcCYP707A67, BcCYP716A41, BcCYP736A53, BcCYP736A54, and BcCYP712F1.

GTs are a superfamily of enzymes in plants. GTs catalyze the transfer of sugar moieties from activated donor molecules to specific acceptor molecules, forming glycosidic bonds. Currently, there are 92 families and some non-classified sequences at the superfamily level http:// www.cazy.org/GlycosylTransferases.html. Glycosylation is one of the major factors that determine the bioactivity and bioavailability of natural plant products, such as flavonoids and terpenoids.

The GTs that are responsible for the glycosylation of natural products are members of the family 1 GTs, the UGTs [23]. In our unique sequences, there were 102 annotated GTs (see additional file 4: Putative P450 and GT genes in the 454 dataset) of which 49 were UGTs. All the GTs were classified into 14 categories according to the GO term assignment (see additional file 6: Classification of the candidate glycosyltransferase/glucosyltransferase genes). Two full-length UGTs were verified by RT-PCR. Four partial UGTs from the 454 dataset and one UGT derived from our previous cDNA library were extended by RACE PCR, resulting in seven fulllength UGTs tentatively named BcUGT1, BcUGT2, BcUGT3, BcUGT4, BcUGT5, BcUGT6, and BcUGT7. The deduced amino acid sequences of these seven UGTs were aligned with some UGTs from other species, and a neighbor-joining tree was constructed based on the alignment (Figure 5). The UGTs from the other plant species were selected based on the BLASTX results for the seven UGTs cloned in the present study. Most of these UGTs were function identified. GmSGT2 (AB473730) and MtGT3 (FJ477891) were identified with functions in the biosynthesis of saponins. AsUGT709A10 (EU496501) and CsUGT4 (GQ221689) may be involved in the formation of triterpenoids and monoterpenoids, respectively, because both were registered in GenBank and relevant unpublished papers. The others were identified as flavonoid GTs. Although the functions of a UGT cannot be deduced from the sequence alignment alone, the functions of BcUGT3 (JF803819) and BcUGT6 (JF803822) were first verified in the course of SS-related UGT identification. Both of these UGTs had high sequence similarities with previously identified terpene GTs.

\section{Expression characteristics of P450s and UGTs}

The pentacyclic triterpenoid SSs are considered to be synthesized using $\beta$-amyrin as a substrate via a series of reactions thought to be catalyzed by P450s and UGTs. MeJA is known to induce the biosynthesis of many secondary metabolites, such as ginsenoside $[47,48]$ and SSs
$[17,49]$. MeJA also up-regulates metabolite-related enzyme genes [50]. The expression of genes involved in SS skeleton biosynthesis has not been studied in MeJA treatment experiments. However, the expression of $\beta$-AS is up-regulated with increased accumulation of SSs in the hairy roots of $B$. falcatum using altered culture media [16]. Therefore, in the present study, MeJA-treated adventitious roots of $B$. chinense were used to investigate the expression of $\beta$ - $A S$, and to screen the putative P450s and UGTs involved in SS biosynthesis. Based on the classifications and read abundances, a total of 14 P450s and 20 UGTs were selected for MeJA inducibility analyses using real-time PCR with actin as the internal reference gene. All selected P450s belonged to the two clans, CYP71 and CYP85, that include all known triterpenes and sterol hydroxylases. As shown in Figure 6, $\beta$ $A S$ expression increased about fourfold in MeJA-treated adventitious roots compared with in the control. All P450s and UGTs assayed were up-regulated by MeJA, whereas the elevated fold expressions of four P450s and eight $U G T$ s were more than that of $\beta$-AS. Therefore, the four P450s (P450-4, P450-5, P450-7, and P450-12) and the eight UGTs (UGT-1, UGT-3, UGT-5, UGT-6, UGT11, UGT-15, UGT-19, and UGT-20) were further assayed for their tissue-specific expression patterns.

Five tissues, roots, stems, leaves, flowers and fruits, were used to analyze the tissue-specific expression patterns of the abovementioned four P450s and eight UGTs. Real-time PCR analysis was performed using $\beta$ tubulin as the internal reference gene. As shown in Figures 7 and 8 , the expression patterns of two P450s (P450-7 and P450-12) and three UGTs (UGT-3, UGT-5, and UGT-15) showed strong similarities with that of $\beta$ AS. P450-12 was obtained from our previous cDNA library. P450-7, UGT-3, UGT-5, and UGT-15 were all obtained from the 454 dataset. The sequence similarity results suggest that UGT-3 and UGT-5 are members of the UGT85 family, whereas UGT-15 belongs to the UGT76 family. These P450s and UGTs can be considered as candidate genes encoding enzymes responsible for SS biosynthesis, and require further study.

\section{Discussion}

The 454 pyrosequencing technology is regarded as a prime choice for novel gene discovery in non-model organisms. In the present study, this technology was applied with the main goal of identifying the P450s and UGTs involved in the biosynthesis of SSs in B. chinense. In previous reports, CYP93E1 from Glycine max was shown to hydroxylate $\beta$-amyrin and sophoradiol with the formation of olean-12-ene-3 $\beta, 24$-diol, and soyasapogenol B, respectively [24]. CYP88D6 from Glycyrrhiza uralensis was identified as a $\beta$-amyrin 11-oxidase [25]. UGT73K1 and UGT71G1 from Medicago truncatula 


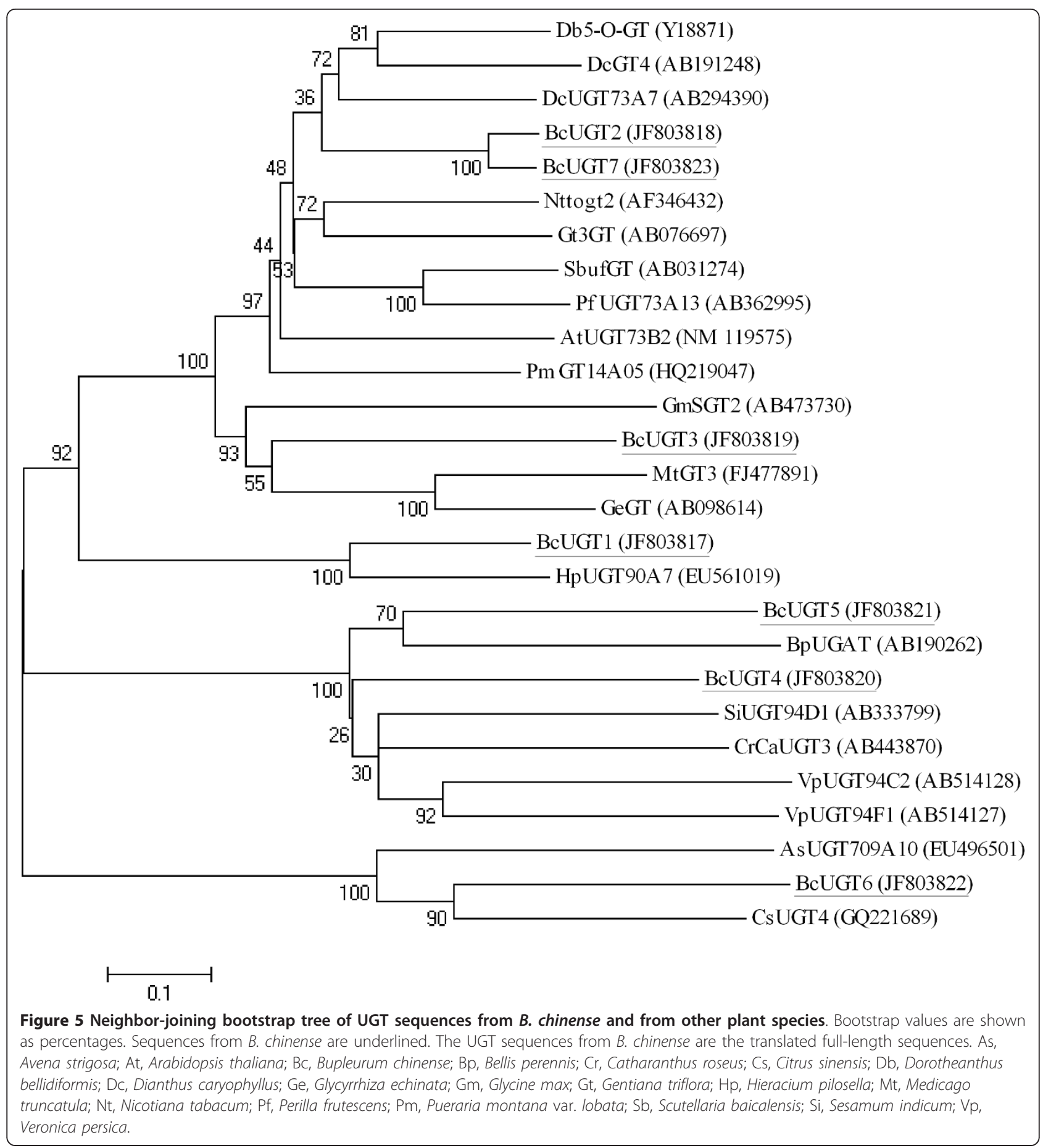

$[26,27]$ and UGT74M1 from Saponaria vaccaria [28] have been identified to be involved in triterpene biosynthesis. Thus far, no P450 or UGT were identified in SS-producing plant species. All known triterpenes and sterol hydroxylases have been classified into the CYP71 and CYP85 clans $[24,25,51,52]$. In our 454 dataset, 114 unique sequences in 8 families and 52 unique sequences in 4 families belong to the CYP71 and CYP85 clans, respectively. Of these 49 were $U G T$ s representing nine families, namely, UGT71, UGT72, UGT73, UGT74, UGT76, UGT84, UGT85, UGT91, and UGT94. Our data provide a promising opportunity for identifying the P450s and UGTs involved in SS biosynthesis. In the present study, 14 unique sequences of P450s and 20 UGTs were screened. Two P450s and three UGTs that may be involved in the biosynthesis of SSs, based on MeJA 


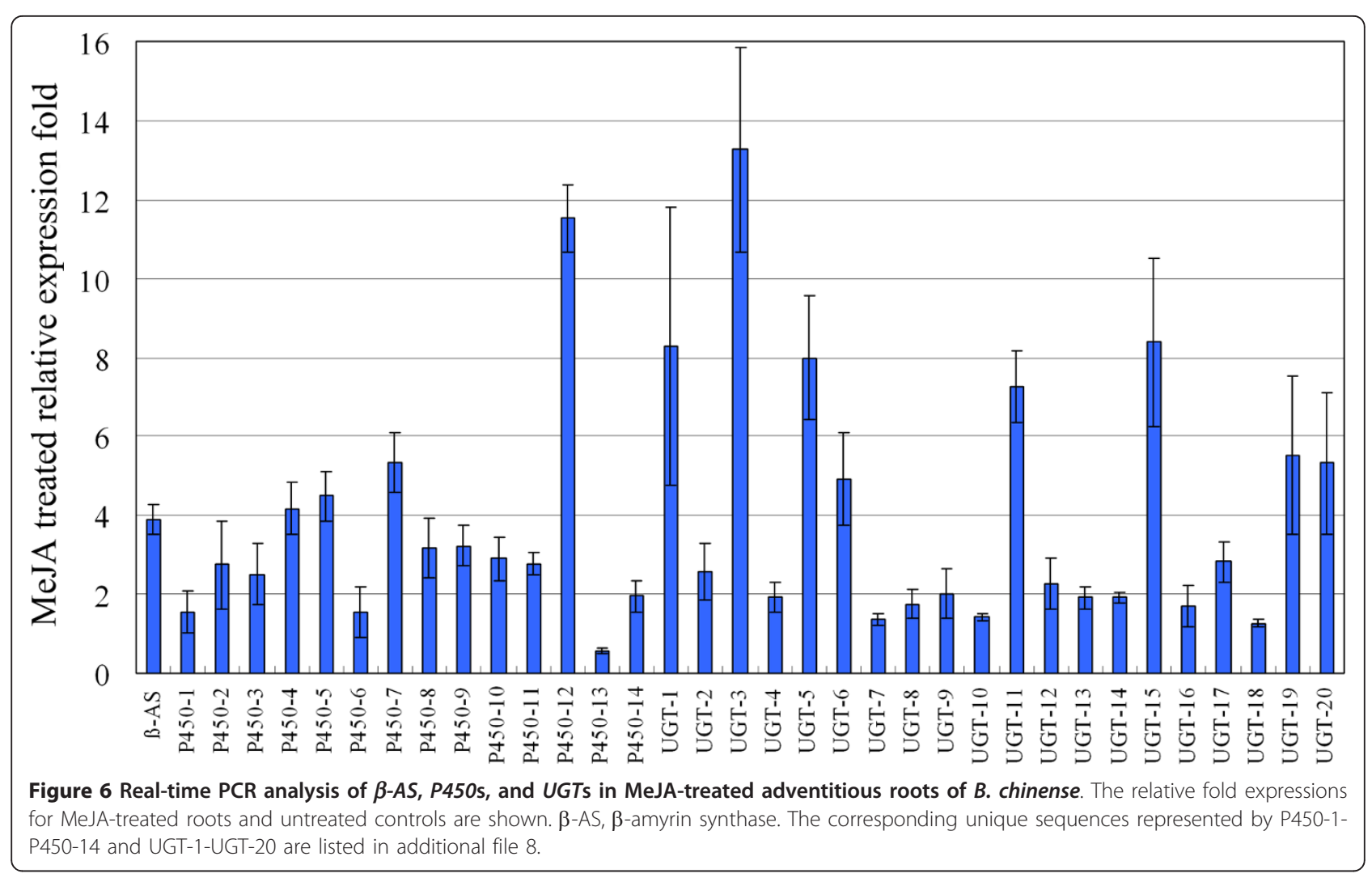

inducibility and tissue-specific expression patterns, were found. They are currently being identified by their heterologous expression in Escherichia coli or yeast, as well as by their overexpression and gene silencing in transgenic B. chinense plants. More candidate SS-related P450s and UGTs may be found among the annotated P450s and UGTs. Along with the identified P450s and $U G T \mathrm{~s}$, our results may also be helpful in revealing the formation mechanism of diverse monomer SSs and in elucidating other saponin biosynthetic pathways.

In the present study, the full-length cDNA clones of seven P450s and seven UGTs were obtained. Two of the P450s belong to the CYP736 family and the other five P450s belong to the CYP82, CYP712, CYP90, CYP707, and $C Y P 716$ families. The catalytic function of the CYP736 family is still unknown. Recent reports have shown that CYP736B in grapes may be involved in the host response to Xylella fastidiosa infection [53]. CYP736A34 in soybean is also highly co-expressed with genes involved in root and Rhizobium-induced nodule development [[54]; review in [55]]. CYP82 and CYP712 are part of the CYP71 clan family. Some members of the CYP82 family were found to mediate plant-specific alkaloid pathways, for example, CYP82E4 and CYP82E5v2 in tobacco were identified with nicotine $N$ demethylase activity [56,57]. Arabidopsis CYP82C2 and CYP82C4 are 8-methoxypsoralen hydroxylases that mediate modifications of toxic furanocoumarin [58]. However, a recent study has shown that CYP82G1 functions in the terpene pathway as a DMNT/TMTT (C11homoterpene (E)-4, 8-dimethyl-1, 3, 7-nonatriene/C16homoterpene (E, E)-4, 8, 12-trimethyltrideca-1, 3, 7, 11tetraene) homoterpene synthase [59]. CYP712 and the CYP93s may catalyze successive steps in the same pathway(s) in different plants [55]. One of the CYP93s, CYP93E1, was found to participate in the triterpene pathway [24]. CYP90, CYP707, and CYP716 are part of the CYP85 clan family. CYP90 is the first family of CYPs required for brassinosteroid synthesis. CYP90Bs, -As, -Ds, and -Cs successively act in the brassinosteroid pathway $[60,55]$. The CYP707s inactivate ABA via 8'hydroxylation to form phaseic acid, and thereby, play a key role in the regulation of ABA-mediated physiological processes [61]. The CYP716s do not have a known function, but their closest non-plant relatives, CYP26As, are involved in the hydroxylation of retinoic acid [55]. Based on sequence similarity, CYP716 was close to CYP725 in the neighbor-joining tree (Figure 4). A previous study using a broader range of plants also showed some overlap in CYP716 and CYP725. This overlap is evidence of the extensive divergence occurring within this subset of genes in the CYP85 clan. CYP725A has been shown to act on taxane diterpenoids [60]. However, it is still unclear whether these two families share 


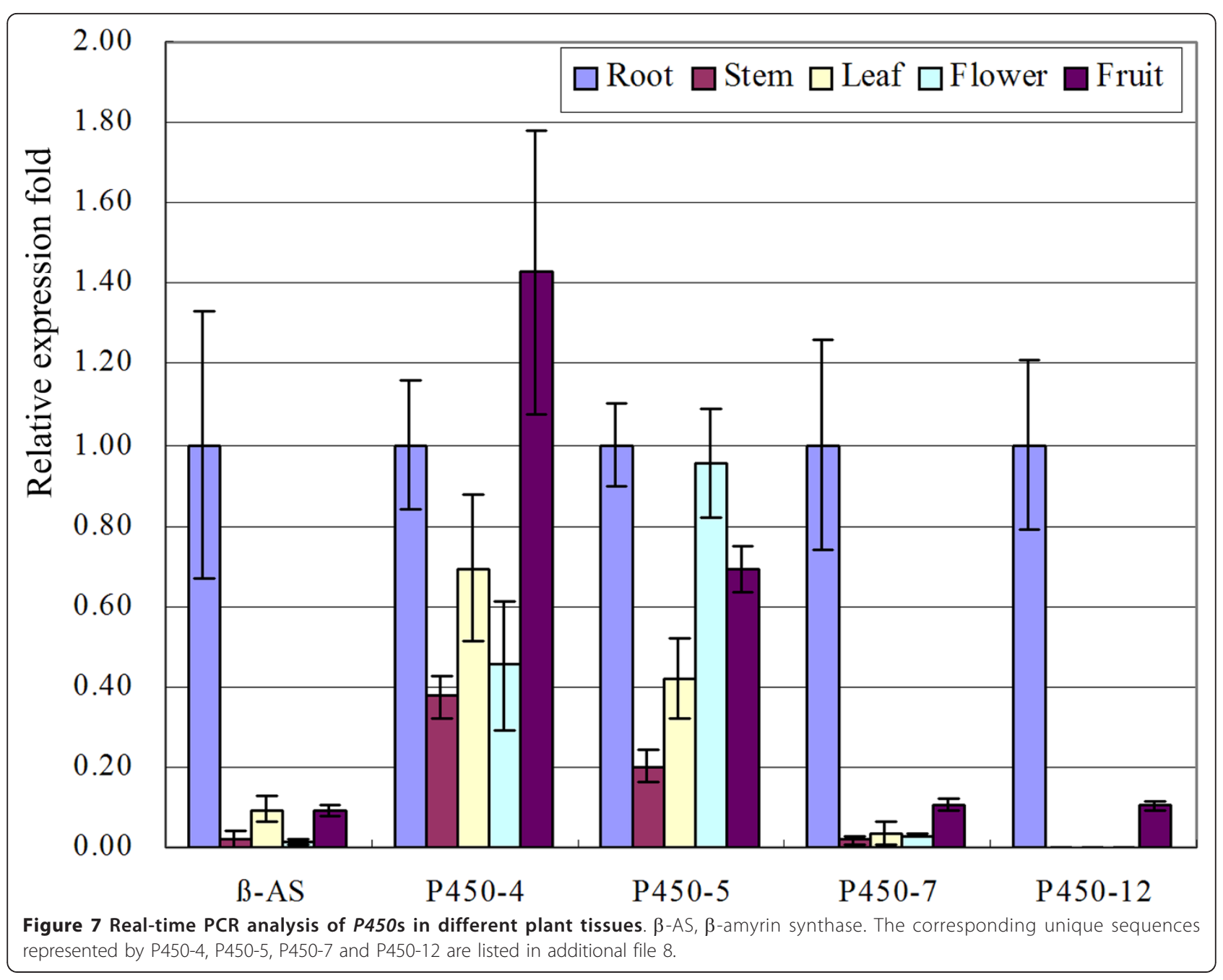

similar functions. The seven $U G T$ s for which full-length cDNAs were generated in the present study have sequence similarities with members of different $U G T$ families. This finding implied that the UGTs identified in the present study may be members of these different UGT families. Based on the neighbor-joining tree (Figure 5), BcUGT3 was found to be close to members of the UGT73 family, in particular to GmSGT2 (UGT73P2), MtGT3 (UGT73F3) and GeGT (UGT73F1); BcUGT6 was close to a UGT709 member. BcUGT2 and BcUGT7 were also close to UGT73 members and to other UGTs without definite family ascriptions. BcUGT1 was close to a UGT90 member. Previous studies $[62,63]$ have indicated that UGT73 and UGT90 belong to the same orthologous group, OG1 [63]. UGT73B2 was shown to exhibit flavonoid 7-O-glucosyltransferase activity [64], UGT73A7 has been reported to exhibit 4, 2', 4', 6'-tetrahydroxy chalcone 4'-glucosyltransferase activity [65], and UGT90A7 was shown to exhibit luteolin 4'/7-O-glucosyltransferase activity [66].
BcUGT4 and BcUGT5 may belong to the UGT94 family because they have sequence similarities with UGT94s. Previous studies have shown that UGT94D1 has UDPglucose: sesaminol 2'-O-glucoside-O-glucosyltransferase activity and UGT94F1 has anthocyanin 3-O-glucoside2"-O-glucosyltransferase activity [67]. Although the definite functions of the seven P450s and seven UGTs from B. chinense identified in the present study still have to be verified by further experiments, the isolation of their full-length cDNAs will be significant for elucidating their biofunctions in the growth and development of $B$. chinense.

The biosynthesis and regulation of bioactive components was the main focus of the present study on $B$. chinense. In addition to SSs isolated from members of the genus Bupleurum that exhibit pharmacological activity, several other groups of secondary metabolites with relevant biological activity have been characterized, for example, polysaccharides with anti-ulcer activity and lignans with anti-proliferative activity [68]. Genes involved 


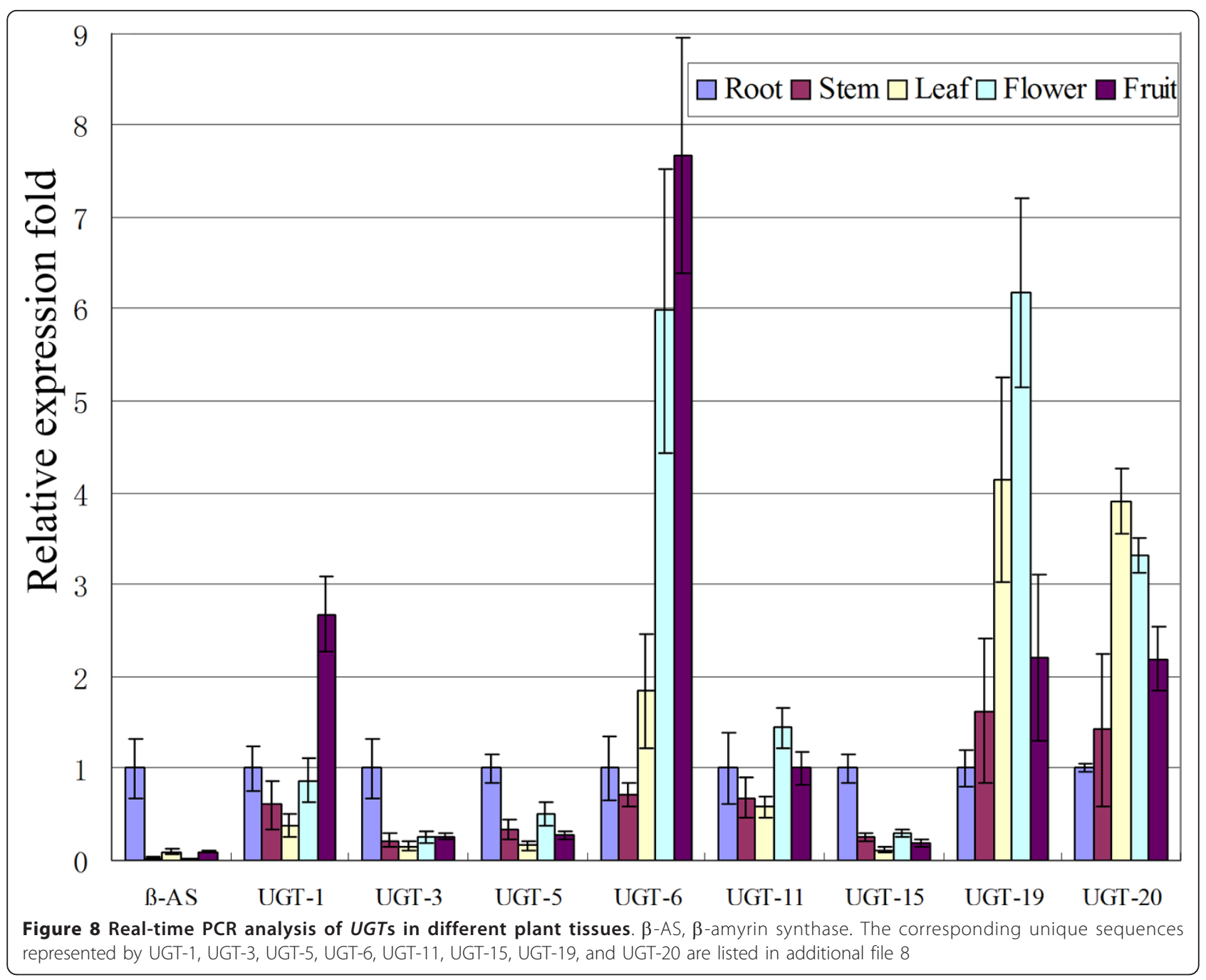

in polysaccharides and lignans were searched for in the present 454 dataset. For example, enzymes encoded by genes related to polysaccharides include $(1,3)$-beta-Dglucan synthase, alpha-1, 6-xylosyltransferase, alpha-(1, 4)-galacturonosyltransferase, xylan 1, 4-beta-xylosidase, etc. [69] and enzymes encoded by the genes related to lignans, are phenylalanine ammonia lyase, cinnamate 4hydroxylase, 4-coumarate-CoA ligase, hydroxycinnamoyl CoA: shikimate/quinate hydroxycinnamoyltransferase, caffeoyl-CoA O-methyltransferase, isoeugenol synthase, and dirigent protein oxidase [70]. Therefore, the present 454 dataset is valuable not only in the exploration of genes involved in SS biosynthesis, but also for the discovery of genes involved in other bioactive secondary metabolites derived from the genus of Bupleurum. Additionally, the agronomical traits of $B$. chinense, such as drought resistance, have been investigated [71,72]. In our 454 dataset, 2, 933 and 3, 280 unique sequences were annotated as related to responses to abiotic or biotic stimulus and to stress, respectively. These annotations were based on the GO terms. These sequence data may be beneficial to further molecular studies on the stress response of $B$. chinense. Further, a total of 415 and 209 unique sequences were annotated with transcription factor activity and signal transduction, respectively. Some of these sequences may play roles in regulating SS metabolism and the stress response. These unique sequences deserve to be cloned and functionally analyzed in future studies.

Currently the 454 pyrosequencing technology is considered as a rapid and economical method to generate high-quantity sequence data. Although a large number of 454 reads were obtained by a quarter run in the present study, nearly a quarter of the ESTs from the Sanger-sequenced 3, 111 clones from our previous cDNA library were not sequenced. The different cDNA libraries (the Sanger sequenced root cDNA library and the 454 sequenced combined cDNA library with roots, 
seeds, and seedlings) and the fact that only the 5' end of the cDNA was sequenced in the Sanger sequencing may explain, to some extent, this difference. In some reports that compared 454 pyrosequencing and traditional Sanger sequencing, bias was found because of differences in the two sequencing methods [73]. Combinations of these two methods have been used in some studies: (1) to generate a high number of good-quality ESTs with improved clustering analysis and with more full-length sequences [73]; (2) to obtain a less biased method for the identification and diversity analysis of microbes and fungi $[74,75]$; and (3) to assemble genome sequences [76]. Recently, Radix bupleuri has aroused global interest, especially in Europe [review in [68]]. However, studies on the molecular biology of Bupleurum are still limited. More transcriptome data will facilitate a deeper understanding and enable the rapid development of Radix bupleuri applications.

\section{Conclusions}

In the present study, a 454 dataset of $B$. chinense was analyzed. These data represent a substantial contribution to the functional genetic studies of $B$. chinense. The identification of enzymes involved in SS biosynthesis may enable the regulation and improvement of SS production levels in plants or in microbial hosts by metabolic engineering. Almost all of the known genes that encoded enzymes involved in the biosynthesis of the SS backbones were explored. A total of 246 P450 and 102 GT unique sequences containing 49 UGTs were obtained. These sequences will be invaluable to the elucidation of the SS biosynthetic pathway and to the exploration of the molecular mechanism underlying the biosynthesis of different monomer SSs. The full-length cDNAs of seven of the P450s and seven of the UGTs from our present 454 dataset and previous Sanger's sequencing data were cloned using the RACE method. This procedure may help in elucidating the functions of the P450s and UGTs. MeJA inducibility and tissue-specific expression pattern experiments were used to screen two P450s and three UGTs that may be involved in SS biosynthesis.

\section{Methods}

\section{Plant material and adventitious root preparation}

The roots of one-year old plants of "Zhongchai No. 1", a mass-selected cultivar of $B$. chinense field-grown in IMPLAD, were collected during the flowering stage because more SSs were found to be contained in the roots during this period [77]. Further, a previous study showed that the SS-d and SS-c content significantly changed in germinating seeds and the content of SS-d peaked on the fourth day [78]. Hence, to acquire a high number of unique candidate genes involved in SS biosynthesis the experimental material used in the present study was 4-day geminating seeds, 12-day seedlings, and the roots of one-year-old Zhongchai No. 1 plants during flowering. The germination was performed in germination boxes under $25^{\circ} \mathrm{C} / 15^{\circ} \mathrm{C}, 8 \mathrm{~L} / 16 \mathrm{D}$ conditions. Before germination, the seeds were soaked for $24 \mathrm{~h}$ in tap water, which was changed four times. After harvest, all materials were immediately frozen in liquid nitrogen and stored in a $-80^{\circ} \mathrm{C}$ freezer for RNA extraction.

To analyze the MeJA inducibility of P450s and UGTs, the adventitious roots of Zhongchai No. 1 were cultivated as described earlier [79]. Similar to the results of our previous experiment, the SS content was approximately doubled in $8 \mathrm{~h}$ MeJA-treated $(200 \mu \mathrm{M}$, dissolved in ethanol) adventitious roots of $B$. chinense, assayed by high performance liquid chromatography [80]. MeJA $(200 \mu \mathrm{M})$ was then added to the cultivation media; an equal quantity of ethanol was used as the control. After $8 \mathrm{~h}$, the treated and control adventitious roots were collected and immediately stored in liquid nitrogen for RNA extraction. For the tissue-specific expression pattern experiments, five tissues (roots, stems, leaves, flowers, and fruits) were collected and similarly restored as described in our previous report [81].

\section{RNA extraction, cDNA library construction and 454 sequencing}

Total RNA was isolated using an RNA purification kit (Norgen Biotek Corp., ON, Canada). RNA purity and degradation were checked on $1 \%$ agarose gels. Equivalent RNAs from roots, germinating seeds, and seedlings were pooled. Approximately $1 \mu \mathrm{g}$ of RNA was reverse transcribed using a Super SMART TM PCR cDNA synthesis kit (Clontech Laboratories, Inc., Mountain View, CA, USA). This kit was used in combination with a modified poly $(\mathrm{T})$ primer to overcome the limitation of long poly $(\mathrm{A} / \mathrm{T})$ tails in $\mathrm{CDNA}$ for the 454 sequencing [15]. Double-stranded (ds) cDNA was synthesized using an Advantage ${ }^{\circledR} 2$ PCR kit (Clontech Laboratories, Inc.) and was then digested overnight with BsgI (New England Biolabs, Ipswich, MA, USA). The ds cDNA was finally purified using a PureLink ${ }^{\mathrm{TM}}$ PCR purification kit (Invitrogen Life Science Technologies, Carlsbad, CA, USA). About $5 \mu \mathrm{g}$ of ds cDNA was sent to the Roche 454 Company (Branford, CT, USA) for pyrosequencing using a GS FLX titanium kit.

\section{The 454 EST assembly and annotation}

A pretreatment process that involved trimming the adapter and poly $(\mathrm{A} / \mathrm{T})$, as well as removing short sequence $(<50 \mathrm{bp})$ and low quality files (quality score threshold $=20$ ) was performed. The Mira 3.0.5 software was used for sequence assembly using the default parameters. Reads that did not fit into a contig were defined 
as singletons. A total of 195, $088 \mathrm{HQ}$ reads assembled in 22, 748 contigs and 1, 289 singletons were finally obtained for further functional annotation with the BLASTX program. The databases KEGG http://www. genome.jp/kegg/, Nr http://www.ncbi.nlm.nih.gov, and UniProt http://www.expasy.ch/sprot were used for the search. GO terms were assigned to the assembled unique genes based on similarities with $A$. thaliana protein sequences (TAIR9, http://www.arabidopsis.org). A cut-off value of $E<1.0^{-10}$ was used in all BLASTX searches. The newly assembled unique genes were compared against the Bupleurum EST/protein encoding sequences in GenBank. The ESTs were derived from a $B$. chinense root cDNA library that was sequenced by our group using an ABI 3730 sequencer [19].

\section{Searching for candidate genes involved SS biosynthesis} The candidate genes HMGR, IPPI, FPS, SQS, SE, $\beta$-AS, $P 450$, and UGT that are known to be involved in the biosynthesis of SSs were searched for within the text of the annotated unique genes based on their gene names and synonyms. The items from different annotation databases that were repeated were manually erased.

\section{Full-length cDNA verification and cloning of P450s and UGTs}

The assembled full-length P450 and UGT cDNA sequences were verified by RT-PCR. Some partial sequences were extended to full length using $5^{\prime}$ and/or 3' RACE. The amino acid sequence alignments of the full-length P450 and UGT cDNAs were created in MEGA 4 using CLUSTALW with default settings. Phylogenetic neighbor-joining trees were constructed and bootstrapped with 1000 iterations in MEGA 4. Corresponding sequences from other plants with the most similarity to each full-length P450 and UGT cDNA (obtained both in our present and previous studies) [19] were identified and downloaded from GenBank. These sequences were used for the alignments and tree constructions.

\section{Real-time PCR analysis}

Actin was chosen as the internal reference gene for the real-time PCR gene expression analysis of MeJA-treated B. kaoi [17]. Similar to our previous report, $\beta$-tubulin was the most suitable reference gene for the real-time PCR analysis of tissue-specific gene expression patterns in B. chinense [81]. According to one of our previous experiments (data unpublished), $E F 1 \alpha$ was also a suitable internal reference gene for real-time PCR analysis in MeJA-treated $B$. chinense. In the present study, the suitability of actin, $\beta$-tubulin, and EF1 $\alpha$ as internal reference genes in the MeJA-treated adventitious roots of $B$. chinense was first determined. Based on the results (see additional file 7: Screening of internal reference genes for real-time PCR analysis of MeJA inducibility), actin was selected as the internal reference gene for the MeJA inducibility experiment. For the tissue-specific expression pattern experiment, $\beta$-tubulin was selected as the internal reference gene based on our previous research [81]. All real-time PCR analyses were performed according to our previous report [81] with the following modifications: the RNA was extracted using an RNA purification kit (Norgen Biotek Corp.); the quantification of cDNA was performed on a NanoDrop ND 2000 spectrophotometer (Thermo Fisher Scientific Inc., Wilmington, DE, USA); and a SYBR ${ }^{\circledR}$ PrimeScript ${ }^{\circledR}$ RT-PCR kit II (Perfect Real-Time; TAKARA Bio Inc., Shiga, Japan) was used for the reverse transcription and real-time PCR. Two-step amplification conditions were used: 3 min at $95^{\circ} \mathrm{C}, 40$ cycles of $30 \mathrm{~s}$ at $95^{\circ} \mathrm{C}$, and $20 \mathrm{~s}$ at $58^{\circ} \mathrm{C}$. For the analyses of the tissue-specific expression patterns, the expression in the root was arbitrarily chosen as the calibrator for each gene. For the MeJA inducibility experiment, the expression of each gene in the control was used as the calibrator. All primers used are listed in additional file 8: The primers used in the present study.

\section{Additional material}

Additional file 1: Summary of the annotation of the 454 assembled unique $B$. chinense sequences. The annotations were obtained by comparing the assembled sequences with sequences from $\mathrm{KEGG}, \mathrm{Nr}$, and UniProt $\left(E<1 \times 10^{-10}\right)$.

Additional file 2: Functional annotations of the 454 unique sequences of $B$. chinense based on GO categories. The annotations were obtained by assigning the 454 assembled unique sequences to the GO categories of molecular function, biological process, and cellular component based on their similarities with $A$. thaliana protein sequences (TAIR9, http://www.arabidopsis.org). A cut-off value of $E<1.0^{-10}$ was used.

Additional file 3: Summary of metabolic pathway assignments of the 454 assembled unique sequences based on KEGG. The numbers of 454 assembled unique sequences that were assigned into different metabolism categories based on KEGG are shown in a bar chart.

Additional file 4: Putative P450 and GT genes in the $\mathbf{4 5 4}$ dataset The 454 assembled unique sequences that were annotated as P450 and GT genes by comparing the assembled sequences with sequences from KEGG, $N$ r, and UniProt $\left(E<1 \times 10^{-10}\right)$ were manual identified and listed.

Additional file 5: Summary of family classification of the annotated P450s from the $\mathbf{4 5 4}$ assembled unique sequences. The number of annotated 454 unique sequences and reads of $B$. chinense encoding P450s that belong to different families and subfamilies are listed. Families belong to the CYP71 clan are shown in red, and families belong to the CYP85 clan are shown in blue.

Additional file 6: Classification of the candidate glycosyltransferase/ glucosyltransferase genes. The assembled 454 unique sequences that were annotated as genes with various glycosyltransferase/ glucosyltransferase activities were classified and listed. The classification was obtained by comparing annotated glycosyltransferase/

glucosyltransferase genes from the 454 dataset with $A$. thaliana protein sequences (TAIR9, http://www.arabidopsis.org). 
Additional file 7: Screening of internal reference genes for real-time PCR analysis of MeJA inducibility. The RNA transcription levels of actin, $\beta$-tubulin, and $E F 1 \alpha$ in the MeJA-treated and control adventitious roots of $B$. chinense were assayed by real-time PCR and are presented as $C t$ values.

Additional file 8: The primers used in the present study. All primers used for full-length CDNA cloning and real-time PCR analysis of P450s and UGTs in the present study are listed.

\section{Acknowledgements}

The authors gratefully acknowledge Huazong Zeng at the Shanghai Sensichip Tech@infor Co. Ltd. for his assistance with bioinfomatics. The present work was supported by the National Natural Science Foundation of China (grant No. 81072994), the Beijing Municipal Natural Science Foundation of China (No. 5102033), and the Research Fund of State Administration of Traditional Chinese Medicine of People's Republic of China (No. 201107011)

\section{Authors' contributions}

CS conceived the study, designed and built the CDNA library, designed the primers for the RACE and the real-time PCR, participated in the data analyses, as well as drafted the manuscript. JZ performed part of the RACE and the real-time PCR experiments. JHW initiated the project, helped conceive the study, and revised the manuscript. SLC helped conceive the study and revised the manuscript. YL and CXX helped collect the sample and analyze the data. JSX performed part of the RACE experiment. YJ helped assay the content of SSs by HPLC in the pre-experiment. ZHG helped build the CDNA library. HJC helped draw the chemical structures. CMY and $\mathrm{ZZ}$ helped conceive the study. YHX helped analyze the gene functions. All authors read and approved the final manuscript.

Received: 6 July 2011 Accepted: 2 November 2011 Published: 2 November 2011

\section{References}

1. Shan RH, She ML: Flora of China. Beijing: Science Press; 1979:55(1):215-295.

2. Chinese Pharmacopoeia Commission: Pharmacopoeia of the People's Republic of China. 2005, , 1: 196-197.

3. Yang ZY, Chao Z, Huo KK, Xie H, Tian ZP, Pan SL: ITS sequence analysis used for molecular identification of the Bupleurum species from northwestern China. Phytomedicine 2007, 14(6):416-423.

4. Yang YY, Tang YZ, Fan CL, Luo HT, Guo PR, Chen JX: Identification and determination of the saikosaponins in Radix bupleuri by accelerated solvent extraction combined with rapid-resolution LC-MS. J Sep Sci 2010, 33:1933-1945

5. Huang $H Q$, Zhang $X$, Lin M, Shen YH, Yan SK, Zhang WD: Characterization and identification of saikosaponins in crude extracts from three Bupleurum species using LC-ESI-MS. J Sep Sci 2008, 31:3190-3201.

6. Ushio Y, Abe H: Inactivation of measles virus and herpes simplex virus by saikosaponin d. Planta Med 1992, 58(2):171-173.

7. Sun Y, Cai TT, Zhou XB, Xu Q: Saikosaponin a inhibits the proliferation and activation of $T$ cells through cell cycle arrest and induction of apoptosis. Int Immunopharmacol 2009, 9(7-8):978-983.

8. Zong Z, Fujikawa-Yamamoto K, Ota T, Guan X, Murakami M, Li A, Yamaguchi N, Tanino M, Odashima S: Saikosaponin b2 induces differentiation without growth inhibition in cultured B16 melanoma cells. Cell Struct Funct 1998, 23(5):265-272.

9. Wong VKW, Zhou H, Cheung SSF, Li T, Liu L: Mechanistic study of saikosaponin-d (Ssd) on suppression of murine T lymphocyte activation. J Cell Biochem 2009, 107(2):303-315.

10. Shyu KG, Tsai SC, Wang BW, Liu YC, Lee CC: Saikosaponin C induces endothelial cells growth, migration and capillary tube formation. Life Sci 2004, 76(7):813-826.

11. Tan LL, Cai X, Hu ZH, Ni XL: Localization and Dynamic Change of Saikosaponin in Root of Bupleurum chinense. J Integr Plant Biol 2008 50(8):951-957.
12. Park KH, Park J, Koh D, Lim Y: Effect of saikosaponin-a, a triterpenoid glycoside, isolated from Bupleurum falcatum on experimental allergic Asthma. Phytother Res 2002, 16:359-363.

13. Lambert E, Faizal A, Geelen D: Modulation of triterpene saponin production: in vitro cultures, elicitation, and metabolic engineering. Appl Biochem Biotechnol 2011, , 164: 220-237.

14. Haralampidis K, Trojanowska M, Osbourn AE: Biosynthesis of triterpenoid saponins in plants. Adv Biochem Eng Biotechnol 2002, 75:31-49.

15. Sun C, Li Y, Wu Q, Luo HM, Sun YZ, Song JY, Lui E, Chen SL: De novo sequencing and analysis of the American ginseng root transcriptome using a GS FLX Titanium platform to discover putative genes involved in ginsenoside biosynthesis. BMC Genomics 2010, 11:262.

16. Kim YS, Cho JH, Ahn J, Hwang B: Upregulation of isoprenoid pathway genes during enhanced saikosaponin biosynthesis in the hairy roots of Bupleurum falcatum. Mol Cells 2006, 22(3):269-274.

17. Chen LR, Chen YJ, Lee CY, Lin TY: MeJA-induced transcriptional changes in adventitious roots of Bupleurum kaoi. Plant Sci 2007, 173:12-24.

18. Liu WY, Peng PH, Lin TY: Cloning and characterization of beta-amyrin synthase from Bupleurum kaoi. 8th International Congress of Plant Molecular Biology. Book of Abstracts, ISPMB 2006, POS-TUE-121, Adelaide, Australia.

19. Sui C, Wei $J H$, Chen SL, Chen HQ, Dong LM, Yang CM: Construction of a full-length enriched cDNA library and analysis of 3111 ESTs from root of Bupleurum chinense DC. Bot Stud 2010, 51(1):16.

20. Sui C, Wei JH, Zhan QQ, Yang CM: Cloning and sequence analysis of squalene synthase gene and cDNA in Bupleurum chinense DC. Acta Horticulturae Sinica 2010, 37(2):283-290, in Chinese.

21. Sui C, Zhan QQ, Wei JH, Chen HQ, Yang CM: Full-length cDNA cloning and sequence analysis of IPPI involved in saikosaponin biosynthesis in Bupleurum chinense DC. Chinese Traditional and Herbal Drugs 2010, 41(7):1178-1184, in Chinese.

22. Mizutani M, Ohta D: Diversification of P450 genes during land plant evolution. Annu Rev Plant Biol 2010, 61:291-315.

23. Wang XQ: Structure, mechanism and engineering of plant natura product glycosyltransferases. FEBS Lett 2009, 583:3303-3309.

24. Shibuya M, Hoshino M, Katsube $Y$, Hayashi H, Kushiro T, Ebizuka Y: Identification of beta-amyrin and sophoradiol 24-hydroxylase by expressed sequence tag mining and functional expression assay. FEBS 2006, 273(5):948-959.

25. Seki H, Ohyama K, Sawai S, Mizutani M, Ohnishi T, Sudo H, Akashi T, Aoki T, Saito K, Muranaka T: Licorice beta-amyrin 11-oxidase, a cytochrome P450 with a key role in the biosynthesis of the triterpene sweetener glycyrrhizin. Proc Natl Acad Sci USA 2008, 105(37):14204-14209.

26. Achnine L, Huhman DV, Farag MA, Sumner LW, Blount JW, Dixon RA: Genomics-based selection and functional characterization of triterpene glycosyltransferases from the model legume Medicago truncatula. Plant $J$ 2005, 41:875-887.

27. Naoumkina MA, Modolo LV, Huhman DV, Urbanczyk-Wochniak E, Tang YH, Sumner LW, Dixon RA: Genomic and coexpression analyses predict multiple genes involved in triterpene saponin biosynthesis in Medicago truncatula. Plant Cell 2010, 22:850-866.

28. Meesapyodsuk D, Balsevich J, Reed DW, Covello PS: Saponin biosynthesis in Saponaria vaccaria. cDNAs encoding beta-amyrin synthase and a triterpene carboxylic acid glucosyltransferase. Plant Physiol 2007, 143(2):959-969.

29. Augustin JM, Kuzina V, Andersen SB, Bak S: Molecular activities, biosynthesis and evolution of triterpenoid saponins. Phytochemistry 2011, 72:435-457.

30. Sawai S, Saito K: Triterpenoid biosynthesis and engineering in plants. Frontier in Plant Science 2011, 2:25.

31. Vincken JP, Heng L, de Groot A, Gruppen H: Saponins, classification and occurrence in the plant kingdom. Phytochemistry 2007, 68:275-297.

32. Ohlrogge J, Benning C: Unraveling plant metabolism by EST analysis. Curr Opin Plant Biol 2000, 3:224-228.

33. Alba R, Fei ZJ, Payton P, Liu Y, Moore SL, Debbie P, Cohn J, D'Ascenzo M, Gordon JS, Rose JKC, Martin G, Tanksley SD, Bouzayen M, Jahn MM, Giovannoni J: ESTs, cDNA microarrays, and gene expression profiling: tools for dissecting plant physiology and development. Plant J 2004, 39:697-714.

34. Goossens A, Rischer $\mathrm{H}$ : Implementation of functional genomics for gene discovery in alkaloid producing plants. Phytochem Rev 2007, 6:35-49. 
35. Jung JD, Park HW, Hahn Y, Hur CG, In DS, Chung HJ, Liu JR, Choi DW: Discovery of genes for ginsenoside biosynthesis by analysis of ginseng expressed sequence tags. Plant Cell Rep 2003, 22(3):224-230.

36. Dhaubhadel S, Farhangkhoee M, Chapman R: Identification and characterization of isoflavonoid specific glycosyltransferase and malonyltransferase from soybean seeds. J Exp Bot 2008, 59(4):981-994.

37. Emrich SJ, Barbazuk WB, Li L, Schnable PS: Gene discovery and annotation using LCM-454 transcriptome sequencing. Genome Res 2007, 17(1):69-73.

38. Morozova $\mathrm{O}$, Marra MA: Applications of next-generation sequencing technologies in functional genomics. Genomics 2008, 92(5):255-264.

39. Hahn DA, Ragland GJ, Shoemaker DD, Denlinger DL: Gene discovery using massively parallel pyrosequencing to develop ESTs for the flesh fly Sarcophaga crassipalpis. BMC Genomics 2009, 10:234.

40. Li Y, Luo HM, Sun C, Song JY, Sun YZ, Wu Q, Wang N, Yao H, Steinmetz A, Chen SL: EST analysis reveals putative genes involved in glycyrrhizin biosynthesis. BMC Genomics 2010, 11:268.

41. Wang W, Wang YJ, Zhang Q, Qi Y, Guo DJ: Global characterization of Artemisia annua glandular trichome transcriptome using 454 pyrosequencing. BMC Genomics 2009, 10:465.

42. Luo HM, Li Y, Sun C, Wu Q, Song JY, Sun YZ, Steinmetz A, Chen SL: Comparison of 454-ESTs from Huperzia serrata and Phlegmariurus carinatus reveals putative genes involved in lycopodium alkaloid biosynthesis and developmental regulation. BMC Plant Biol 2010, 10:209.

43. Zagrobelny M, Scheibye-Alsing K, Jensen NB, Moller BL, Gorodkin J, Bak S: 454 pyrosequencing based transcriptome analysis of Zygaena filipendulae with focus on genes involved in biosynthesis of cyanogenic glucosides. BMC Genomics 2009, 10:574.

44. Okada K, Kasahara H, Yamaguchi S, Kavaide H, Kamiya Y, Nojiri H, Yamane $\mathrm{H}$ : Genetic evidence for the role of isopentenyl diphosphate isomerases in the mevalonate pathway and plant development in Arabidopsis. Plant Cell Physiol 2008, 49:604-616.

45. Phillips MA, D'Auria JC, Gershenzon J, Pichersky E: The Arabidopsis thaliana type I isopentenyl diphosphate isomerases are targeted to multiple subcellular compartments and have overlapping functions in isoprenoid biosynthesis. Plant Cell 2008, 20:677-696.

46. Nelson DR, Koymans L, Kamataki T, Stegeman JJ, Feyereisen R, Waxman DJ, Waterman MR, Gotoh O, Coon MJ, Estabrook RW, Gunsalus IC, Nebert DW: P450 superfamily: update on new sequences, gene mapping, accession numbers and nomenclature. Pharmacogenetics 1996, 6(1):1-42.

47. Thanh NT, Murthy HN, Yu KW, Hahn EJ, Paek KY: Methyl jasmonate elicitation enhanced synthesis of ginsenoside by cell suspension cultures of Panax ginseng in 5-I balloon type bubble bioreactors. Appl Microbiol Biotechnol 2005, 67(2):197-201.

48. Kim YS, Hahn EJ, Murthy HN, Paek KY: Adventitious root growth and ginsenoside accumulation in Panax ginseng cultures as affected by methyl jasmonate. Biotechnol Lett 2004, 26(21):1619-1622.

49. Aoyagi H, Kobayashi Y, Yamada K, Yokoyama M, Kusakari K, Tanaka H: Efficient production of saikosaponins in Bupleurum falcatum root fragments combined with signal transducers. Appl Microb Biotech 2001, 57(4):482-488.

50. Zhao CL, Cui XM, Chen YP, Liang QA: Key enzymes of triterpenoid saponin biosynthesis and the induction of their activities and gene expressions in plants. Nat Prod Commun 2010, 5:1147-1158.

51. Fujita S, Ohnishi T, Watanabe B, Yokota T, Takatsuto S, Fujioka S, Yoshida S, Sakata K, Mizutani M: Arabidopsis CYP90B1 catalyses the early C-22 hydroxylation of C27, C28 and C29 sterols. Plant J 2006, 45(5):765-774.

52. Shimada Y, Fujioka S, Miyauchi N, Kushiro M, Takatsuto S, Nomura T, Yokota T, Kamiya Y, Bishop GJ, Yoshida S: Brassinosteroid-6-oxidases from Arabidopsis and tomato catalyze multiple C- 6 oxidations in brassinosteroid biosynthesis. Plant Physiol 2001, 126(2):770-779.

53. Cheng DW, Lin H, Takahashi Y, Walker MA, Civerolo EL, Stenger DC: Transcriptional regulation of the grape cytochrome P450 monooxygenase gene CYP736B expression in response to Xylella fastidiosa infection. BMC Plant Biol 2010, 10:135.

54. Guttikonda SK, Trupti J, Bisht NC, Chen H, An YQ, Pandey S, Xu D, Yu O: Whole genome co-expression analysis of soybean cytochrome P450 genes identifies nodulation-specific P450 monooxygenases. BMC Plant Biol 2010, 10:243.

55. Nelson D, Werck-Reichhart D: A P450-centric view of plant evolution. Plant J 2011, 66:194-211.
56. Gavilano LB, Siminszky B: Isolation and characterization of the cytochrome P450 gene CYP82E5V2 that mediates nicotine to nornicotine conversion in the green leaves of tobacco. Plant Cell Physiol 2007, 48:1567-1574.

57. Chakrabarti M, Bowen SW, Coleman NP, Meekins KM, Dewey RE, Siminszky B: CYP82E4-mediated nicotine to nornicotine conversion in tobacco is regulated by a senescencespecific signaling pathway. Plant Mol Biol 2008, 66:415-427.

58. Kruse T, Ho K, Yoo HD, Johnson T, Hippely M, Park JH, Flavell R, Bobzin S: In planta biocatalysis screen of P450s identifies 8-methoxypsoralen as a substrate for the CYP82C subfamily, yielding original chemical structures. Chem Biol 2008, 15:149-156.

59. Lee S, Badieyan S, Bevan DR, Herde M, Gatz C, Tholl D: Herbivore-induced and floral homoterpene volatiles are biosynthesized by a single P450 enzyme (CYP82G1) in Arabidopsis. Proc Natl Acad Sci USA 2010, 107(49):21205-21210.

60. Nelson DR, Ming R, Alam M, Schuler MA: Comparison of cytochrome P450 genes from six plant genomes. Tropical Plant Biol 2008, 1:216-235.

61. Mizutani M, Todoroki Y: ABA 8'-hydroxylase and its chemical inhibitors. Phytochem Rev 2006, 5:385-404.

62. Paquette $\mathrm{S}$, Moller $\mathrm{BL}, \mathrm{Bak} \mathrm{S}$ : On the origin of family 1 plant glycosyltransferases. Phytochemistry 2003, 62:399-413.

63. Yonekura-Sakakibara K, Hanada K: An evolutionary view of functional diversity in family 1 glycosyltransferases. Plant J 2011, 66:182-193.

64. Willits MG, Giovanni M, Prata RT, Kramer CM, De Luca V, Steffens JC, Graser G: Bio-fermentation of modified flavonoids: an example of in vivo diversification of secondary metabolites. Phytochemistry 2004, 65:31-41.

65. Togami J, Okuhara H, Nakamura N, Ishiguro K, Hirose C, Ochiai M, Fukui Y, Yamaguchi M, Tanaka Y: Isolation of cDNAs encoding tetrahydroxychalcone 2'-glucosyltransferase activity from carnation, cyclamen, and catharanthus. Plant Biotechnol 2011, 28:231-238.

66. Witte S, Moco SW, Vervoort J, Matern U, Martens S: Recombinant expression and functional characterisation of regiospecific flavonoid glucosyltransferases from Hieracium pilosella L. Planta 2009, 229(5):1135-1146.

67. Ono E, Ruike M, Iwashita T, Nomoto K, Fukui Y: Co-pigmentation and flavonoid glycosyltransferases in blue Veronica persica flowers. Phytochemistry 2010, 71(7):726-735.

68. Ashour ML, Wink M: Genus Bupleurum: a review of its phytochemistry, pharmacology and modes of action. J Pharm Pharmcol 2011, 63:305-321.

69. Geshi N, Petersen BL, Scheller HV: Toward tailored synthesis of functional polysaccharides in plants. Ann NY Acad Sci 2010, 1190:50-57.

70. Kim HJ, Ono E, Morimoto K, Yamagaki T, Okazawa A, Kobayashi A, Satake H: Metabolic engineering of lignan biosynthesis in Forsythia cell culture. Plant Cell Physiol 2009, 50(12):2200-2209.

71. Zhu ZB, Liang ZS, Han RL, Wang X: Impact of fertilization on drought response in the medicinal herb Bupleurum chinense DC Growth and saikosaponin production. Ind Crops Prod 2009, 29(2-3):629-633.

72. Zhu ZB, Liang ZS, Han RL: Saikosaponin accumulation and antioxidative protection in drought-stressed Bupleurum chinense DC Plants. Environ Exp Bot 2009, 66(2):326-333.

73. Swarbreck SM, Lindquist EA, Ackerly DD, Andersen GL: Analysis of leaf and root transcriptomes of soil-grown Avena barbata plants. Plant Cell Physiol 2011, , 52(2): 317-332.

74. Tedersoo L, Nilsson RH, Abarenkov K, Jairus T, Sadam A, Saar I, Bahram M, Bechem E, Chuyong G, Köljalg U: 454 Pyrosequencing and Sanger sequencing of tropical mycorrhizal fungi provide similar results but reveal substantial methodological biases. New Phytol 2010, 188(1):291-301.

75. Edgcomb V, Orsi W, Bunge J, Jeon S, Christen R, Leslin C, Holder M, Taylor GT, Suarez P, Varela R, Epstein S: Protistan microbial observatory in the Cariaco Basin, Caribbean. I. Pyrosequencing vs Sanger insights into species richness. ISME J 2011, 1-13.

76. Woycicki R, Przybecki Z: Pyrosequencing/Sanger plant genome assembly (limitations, problems and solutions) - on the way to cucumber (Cucumis sativus L. cv. Borszczagowski) draft genome sequence publishing. Nature Precedings 2010.

77. Yang CM, Wei JH, Cheng HZ, Chen SL, Ma FJ, Huang ZW: Study on the content undulation of saikosaponin in Bupleurum chinense DC. J Chin Med Mat 2006, 29:316-318. 
78. Minami M, Sugino M, Hata K, Hasegawa C, Ohe C: Effects of light and temperature on germination rate development of embryo and change of saikosaponins content during germinating process in the seeds of Bupleurum falcatum. Nat med 1997, 51(1):40-44.

79. Kusakari K, Yokoyama M, Inomata S: Enhanced production of saikosaponins by root culture of Bupleurum falcatum L. using two-step control of sugar concentration. Plant Cell Rep 2000, 19(11):1115-1120.

80. Zhan QQ, Jin Y, Wei JH, Zhang J, Sui C: Cultivation of adventitious roots and effect of methyl jasmonate on its saikosaponins contents for Bupleurum chinense DC. Letters in Biotechnology 2011, 22(1):57-60, in Chinese.

81. Dong LM, Sui C, Liu YJ, Yang Y, Wei JH, Yang YF: Validation and application of reference genes for quantitative gene expression analyses in various tissues of Bupleurum chinense. Mol Biol Rep 2011.

doi:10.1186/1471-2164-12-539

Cite this article as: Sui et al.: Transcriptome analysis of Bupleurum chinense focusing on genes involved in the biosynthesis of saikosaponins. BMC Genomics 2011 12:539.

\section{Submit your next manuscript to BioMed Central} and take full advantage of:

- Convenient online submission

- Thorough peer review

- No space constraints or color figure charges

- Immediate publication on acceptance

- Inclusion in PubMed, CAS, Scopus and Google Scholar

- Research which is freely available for redistribution

Submit your manuscript at www.biomedcentral.com/submit 